\title{
Biomechanical and Psychophysical Evaluation of Operating Loads in Vehicular Driving
}

\author{
Yoshiyuki Tanaka*, Mitsuhiro Narusue ${ }^{\dagger}$, Hiroaki Matsubara ${ }^{\dagger}$, Toshio Tsuji ${ }^{\ddagger}$, \\ Tomonori Ohtsubo $^{\dagger}$, Hiroshi Okiyama ${ }^{\dagger}$, Matsuhiro Tanaka ${ }^{\dagger}$ and Shunichiro Miyawaki ${ }^{\dagger}$ \\ ${ }^{*}$ Graduate School of Engineering, Nagasaki University, Nagasaki, JAPAN \\ ${ }^{\dagger}$ Mazda Motor Corporation, Hiroshima, JAPAN \\ ${ }^{\ddagger}$ Graduate School of Engineering, Hiroshima University, Hiroshima, JAPAN \\ Email: ytnk@nagasaki-u.ac.jp
}

\begin{abstract}
The present paper proposes an evaluation method of operation loads in vehicular driving, such as the joint-load, the seat pressure, and the perceiving force, based on biomechanical and psychophysical evidences to assist the human-centered design of driving interfaces. The prototype simulator is developed by means of a big experimental data of human motor properties and force-perception properties related with driving operations. The usefulness of the proposed methodology is then demonstrated through a set of simulation experiments in the case of the curve traveling situation.
\end{abstract}

Keywords-Human-centered design, driving interface, force perception properties, human impedance properteis

\section{INTRODUCTION}

A human driver regulates his/her posture with regard to the position of the driving seat and driving interfaces, such as a steering wheel and a gas pedal, in the preparation for driving. He/she then maneuvers the interfaces actively according to the driving situation while enduring operating loads for body weight, reaction force from interfaces and vehicle motion. However, uncomfortable layout and dynamic properties of driving interfaces, as well as unexpected vehicle behavior, have significant impact on driving posture and body movements which might cause exceeding fatigue and lead to an erroneous operation. It will be helpful to incorporate biological evidences on human sensory-motor properties into the layout design of the interior package and the control system of driving interfaces for further improving the driving feel and performance.

There have been many studies that analyzed dynamic characteristics of human movements in vehicular driving. For instance, Park et al.[1] measured stiffness during multi-joint movements of the lower limb involved in the operation of braking. They also designed brake systems considering measured results, and also performed the perception test on the condition of road surface with the braking simulator. Hirao et al. [2] estimated muscle force and joint load depending on the sitting posture using a musculoskeletal model. They reported that the driving posture to reduce fatigue closes to the neutral posture under weightlessness. Tanaka et al. [3][4] developed a biomechanical evaluation method based on force manipulability incorporated human joint-motor properties, which can estimate the spatial characteristics of end-point force generated by the limb in isometric. They applied into the humancentered layout design of a vehicle interior including driving interfaces in the development of an actual vehicle. Although these previous studies evaluated human motor characteristics for the evaluation and design of a driving interface, human sensory characteristics were not considered at all.

On the other hand, there have been a few research studies examined perceptual characteristics during the operations of driving interface. For an example, Abbink et al. [5][6] examined the desirable vibration pattern by the pedal for an alert signal according to the material of shoes and the differential threshold of force perception at the foot. Wang et al.[7] measured driving posture during pedal operation and explored the relationship between comfort rating of clutch pedal operation and biomechanical parameters such as joint angle, moment, and work. Tanaka et al.[8] proved human forceperception characteristics in operating the pedal through a set of perception tests and biomechanical analysis. They reported that the force-perception characteristics is much affected by the contact condition between foot and pedal pad that changes in pedaling operation although dynamic characteristics of the pedal were the same. Similarly Takemura et al.[9] presents the initial experimental analysis of force-perception characteristics relating to steering operation. Furthermore Tanaka et al. [8] proposed the basic methodology of perceptual design of reaction-force for a driving interface. These experimental studies, however, were carried out under the specified posture, and did not discuss an estimation procedure for a certain driving posture.

Thus, the aim of this research paper is to develop a novel computer-aided simulation system that enables to compute and evaluate physical and perceptual loads during maneuvering the driving interfaces based on human sensory-motor properties.

This paper is organized as follows: Section II proposes a biomechanical and psychophysical evaluation method of operating loads in driving, and describes a driver-vehicle model involved in human sensory-motor characteristics and vehicle characteristics. Section III develops the prototyped simulation system that has the data of human sensory-motor properties and vehicle properties measured in advance. In Section IV, the effectiveness and potential of the proposed strategy is verified through simulation experiments. 


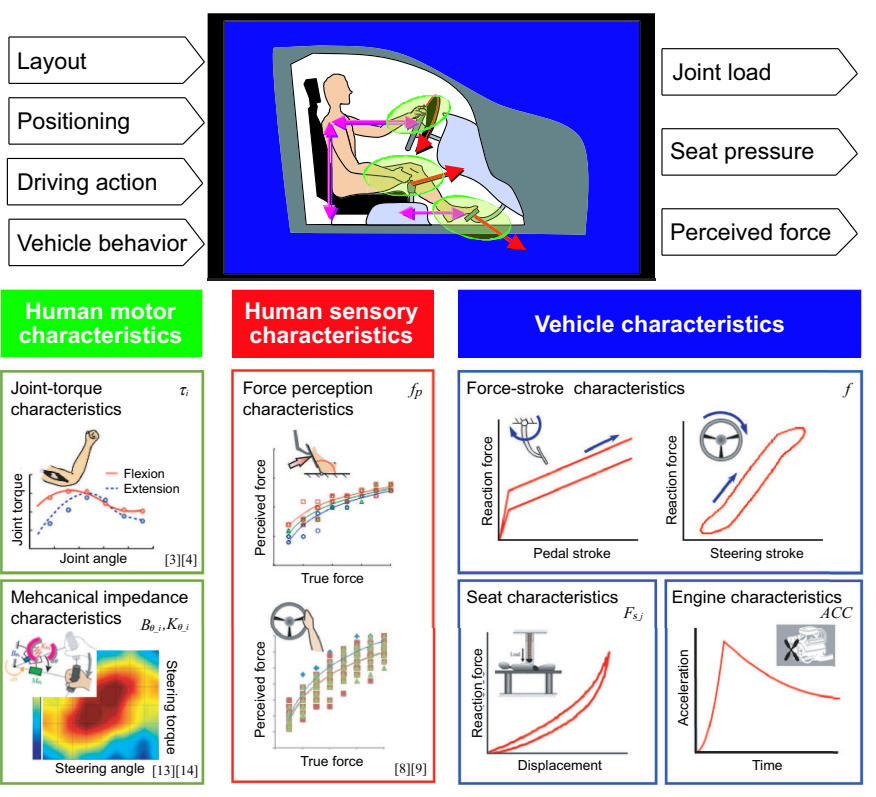

Fig. 1. An illustrative overview of the proposed evaluation methodology.

\section{Evaluation Method}

The conceptual illustration of the proposed evaluation methodology is shown in Fig. 1. The main feature is to evaluate operating loads in driving, such as limb's joint-load, seat-pressure and perceiving resistance force, by means of a database of biomechanical and psychophysical properties of normal humans measured in the experiments.

\section{A. Dynamics of human driver and vehicle interface}

Dynamic behaviors of the human limb is computed by the following equation as

$M_{i} \ddot{\theta}_{i}+h_{i}\left(\dot{\theta}_{i}, \theta_{i}\right)+g_{i}\left(\theta_{i}\right)+B_{\theta} \_i \dot{\theta}_{i}+K_{\theta} \_i\left(\theta_{i}-\theta_{i}^{v}\right)=\tau_{i}$

where $i \in\{$ arm, leg $\}, \theta_{i}, \theta_{i}^{v} \in \Re^{n}$ denote the driving posture (joint angle) and the initial posture, respectively, $\tau_{i} \in \Re^{n}$ is the joint torque, $M_{i} \in \Re^{n \times n}$ is the non-singular inertia matrix, $h_{i} \in \Re^{n}$ represents the non-linear terms including the joint torque due to the Coriolis effect and centrifugal force, $g_{i} \in \Re^{n}$ is the joint torque caused by gravity, $B_{\theta \_i} \in \Re^{n \times n}$ and $K_{\theta \_i} \in$ $\Re^{n \times n}$ are the joint-viscosity matrix and joint-stiffness matrix. Dynamic properties of human movements are much influenced by the arm/leg posture and muscle activation level. However, to measure viscoelastic properties for the joint with multiple degree-of-freedom is almost impossible. Considering this fact, the viscoelastic properties of limb's joint are computed [10] by

$$
\begin{gathered}
B_{\theta \_i}=J_{i}(\theta)_{i}^{T} B_{e_{-} i} J_{i}\left(\theta_{i}\right), \\
K_{\theta \_i}=J_{i}\left(\theta_{i}\right)^{T} K_{e \_i} J_{i}\left(\theta_{i}\right)+\frac{\partial J_{i}\left(\theta_{i}\right)}{\partial \theta_{i}} F_{e x t \_i},
\end{gathered}
$$

where $B_{e_{-} i}, K_{e_{-} i} \in \Re^{m \times m}$ are the viscosity and stiffness matrices at the end-point, respectively, and $F_{\text {ext } i}$ is the external force exerted to the end-point, $J_{i}\left(\theta_{i}\right) \in \bar{\Re}^{m \times n}$ is the Jacobian matrix on the end-point position $x_{i} \in \Re^{m}$ with respect to $\theta_{i}$.

A set of 1-DOF spring-damper systems is arranged between the driver's skin and the seat surface, and the interaction force $F_{s \_j}$ at the j-th point is calculated by

$$
F_{s \_j}=B_{s \_j} \dot{x}_{j}+K_{s \_j} x_{j}
$$

where $x_{j}$ denotes the displacement of seat surface from an original form, $K_{s \_j}$ and $B_{s_{-j} j}$ denote the seat stiffness and viscosity parameters, respectively. This study assumes that a driving interface device is impedance-controlled to produce various resistance force/torque that will much affect to the operational feeling for human driver. For an example, dynamics of the pedal can be given by

$$
\begin{aligned}
\tau_{\phi} & =B_{\phi} \dot{\phi}+K_{\phi} \phi \\
& =f l_{d}
\end{aligned}
$$

where $\tau_{\phi}$ is the reaction torque against the driver's operational force $f$ thatis perpendicular to the pedal pad, $l_{d}$ is the distance between the rotational center of pedal and the contact point to the foot, and $K_{\phi}$ and $B_{\phi}$ are stiffness and viscosity around the pedal rotation axis, respectively.

\section{B. Biomechanical index and Psychophysical model}

A human driver would have to withstand his/her own weight and inertia force to maintain suitable driving posture according to driving situations, while he/she would generate operational force for steering and pedaling to control the vehicle as he/she hopes. It may be desirable to reduce joint-load in driving as much as possible. Therefore the evaluation index $E_{\text {load }}^{i}$ in maintaining posture against external forces is designed based on human joint-torque properties [3][4] as

$$
E_{\text {load }}^{i}=\sum_{l}^{R, L}\left(\sum_{k=1}^{n}{ }^{l} w_{(k)} \frac{{ }^{l} \tau_{i}^{(k)}\left({ }^{l} \theta_{i}^{(k)}\right)}{{ }^{l} \tau_{i \_ \text {max }}^{(k)}\left({ }^{l} \theta_{i}^{(k)}\right)}\right),
$$

where $l \in\{R:$ Right, L:Left $\}, k$ is the joint number, ${ }^{l} w_{(k)}$ is the weighting factor, $\tau_{i}^{(k)}$ expresses the torque calculated with Eq. (1), and $\tau_{i}^{(k)}$ max represents the maximum joint torque that a normal human could produce at the joint angle ${ }^{l} \theta_{i}^{(k)}$. Note that the smaller value of $E_{\text {load }}^{i}$ represents the smaller joint load for a target driving situation.

On the other hand, it is well-known that the relationship between the magnitude of a physical stimulus and the intensity/strength that people would feel follows the Fechner's low. This well-known psychophysical evidence has been also confirmed for the reaction force in the case of the pedaling operation by the leg [13] and the steering operation by the arms [14]. Accordingly, the relationship between the perceived magnitude of an operational force $f_{p}$ and the reaction force $f$ at the control input (stroke angle) $\eta$ is successfully modeled by a logarithmic curve as

$$
f_{p}(\eta)=a_{\eta} \log f(\eta)+b_{\eta}
$$

Where $a_{\eta}, b_{\eta}$ are the fitting parameters with experimental data measured at each stroke input $\eta$. Consequently the perceiving force $f_{p}$ that a driver would feel in the operation of a driving interface is estimated by a set of logarithmic curves as shown in Fig. 2. 




Fig. 2. The conversion of operating force to perceiving force.

TABLE I. PhySICAL DATA OF A HUMAN MODEL.

\begin{tabular}{c|ccccc}
\hline Segment & $\begin{array}{c}\text { Length } \\
{[\mathrm{m}]}\end{array}$ & $\begin{array}{c}\text { Mass } \\
{[\mathrm{kg}]}\end{array}$ & $\begin{array}{c}M x \\
{\left[\mathrm{~kg} \cdot \mathrm{m}^{2}\right]}\end{array}$ & $\begin{array}{c}M y \\
{\left[\mathrm{~kg} \cdot \mathrm{m}^{2}\right]}\end{array}$ & $\begin{array}{c}M z \\
{\left[\mathrm{~kg} \cdot \mathrm{m}^{2}\right]}\end{array}$ \\
\hline Head & 0.14 & 4.1552 & 0.0209 & 0.0186 & 0.0126 \\
Neck & 0.10 & 1.2493 & 0.1770 & 0.1770 & 0.1175 \\
Upper body & 0.27 & 17.3511 & 0.1831 & 0.2407 & 0.1624 \\
Lower body & 0.21 & 12.3082 & 0.0984 & 0.1219 & 0.1015 \\
Upper arm & 0.32 & 1.7863 & 0.0022 & 0.0118 & 0.0122 \\
Forearm & 0.25 & 1.0464 & 0.0009 & 0.0052 & 0.0053 \\
Hand & 0.06 & 0.3829 & 0.0002 & 0.0007 & 0.0006 \\
Thigh & 0.46 & 8.3320 & 0.0243 & 0.1224 & 0.1280 \\
Shank & 0.49 & 3.8247 & 0.0062 & 0.0688 & 0.0681 \\
Foot & 0.16 & 0.3771 & 0.0015 & 0.0001 & 0.0015 \\
\hline
\end{tabular}

\section{PROTOTYPE SIMULATOR FOR THE EVALUATION OF OPERATING LOADS}

A prototype simulator is developed by the combination of the mechanical analysis software (ADAMS, Mechanical Dynamics Inc.) and the numerical computation software (MATLAB, Mathworks Inc.) as shown in Fig. 3, where the numbers around the human driver model denote the degree-of-freedom for each joint.

\section{A. Setting for driver model}

In this paper the physical properties of body parts, such as weight and moment inertia in the matrix $M$, are determined based on the data in the literature [12] as presented in Table I. The maximum torque at each joint of human limbs, which varies in the joint angle and the rotational direction, is calculated with the dataset reported in the literature [3][4].

The measured data of human arm impedance around the steering axis, $B_{\theta}$ arm and $K_{\theta}$ arm , and human leg impedance in the vertical direction of the pedal pad, $B_{\theta}$ leg and $K_{\theta}$ leg, are shown in Figs. 4 (a) and (b), respectively [13][14]. Note that the viscosity and stiffness matrices, $B_{e_{-} i}$ and $K_{e_{-} i}$, are calculated with Eqs. (2) and (3).

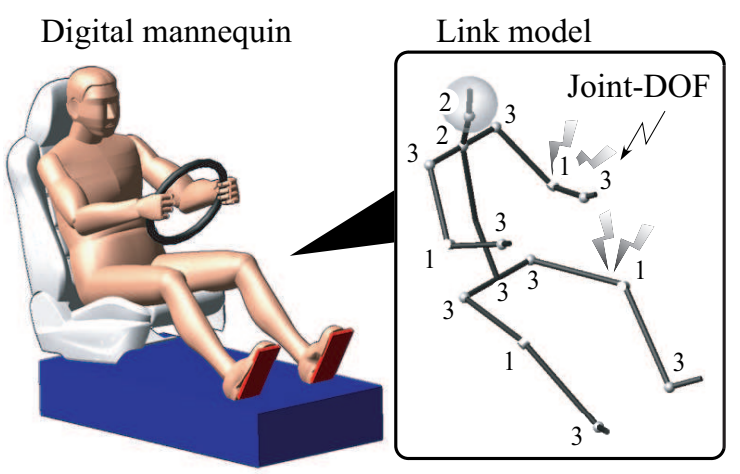

(a)

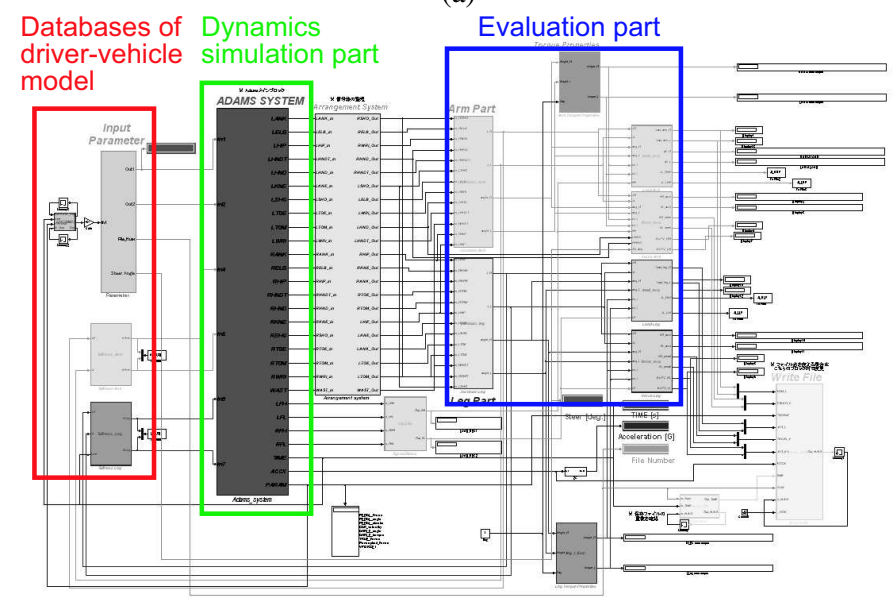

(b)

Fig. 3. An outline of the developed prototype simulator.

TABLE II. PARAMETER VALUES OF LOGARITHMIC CURVES FOR FORCE PERCEPTION.

\begin{tabular}{|c|c|c|c|c|c|}
\hline $\begin{array}{r}\text { Pedal angle } \\
\phi \text { [deg.] }\end{array}$ & $a$ & $b$ & $\begin{array}{l}\text { Steering angle } \\
\theta_{\text {steer }}[\mathrm{deg} .]\end{array}$ & $a$ & $b$ \\
\hline 115 & 50.93 & -136.71 & 0 & 11.21 & -13.42 \\
\hline 130 & 45.08 & -102.74 & 60 & 12.34 & -16.89 \\
\hline 145 & 40.55 & -75.39 & 120 & 11.54 & -14.76 \\
\hline
\end{tabular}

The relationship between reaction force and perceived force with relation to the control input for the driving interface is provided in Fig. 4(c) [8][9]. Note that the parameter values of the derived logarithmic curve for the pedal angle were set as $a_{\text {pedal }}=59.188, b_{\text {pedal }}=-174.68$, and for the steering angle were set as $a_{\text {steer }}=11.285, b_{\text {steer }}=-13.419$ by following the procedure presented in II-B.

\section{B. Setting for vehicle model}

Fig. 5(a) shows the temporal changes of vehicle acceleration according to the stroke angle of the gas pedal, where the green lines denote the measured data at 20 and $50 \%$ strokes and the blue lines the simulated data. The engine performance 



(a)

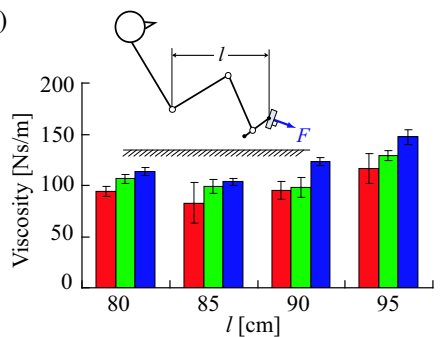

(b)
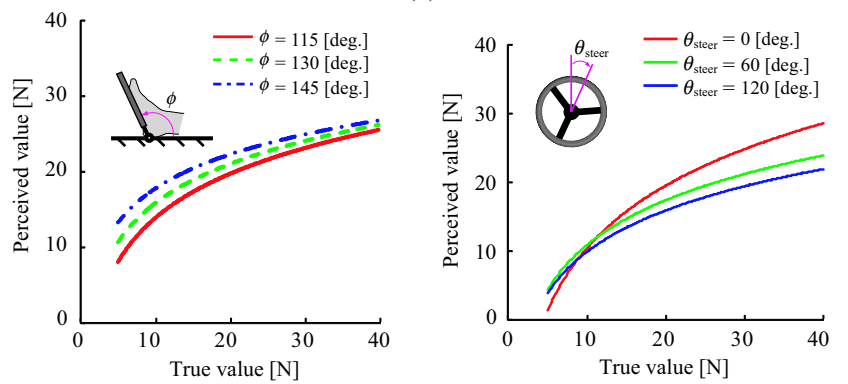

(c)

Fig. 4. A set of human impedance properties and human force perception properties.

characteristics, $A C C(t, \phi)$, was modeled as follows:

$A C C(t, \phi)= \begin{cases}\frac{A C C_{\max }(\phi)}{1.56^{2}}\left(t-t_{\text {rise }}\right)^{2} & \left(t<t_{a}\right) \\ 0.0532\left(t-t_{a}\right)^{2}-0.6988\left(t-t_{a}\right)+ & \left(t \geq t_{a}\right),\end{cases}$

where the peak of vehicle acceleration $A C C_{\max }$ is calculated by

$$
A C C_{\max }(\phi)=-0.002 \phi^{2}+0.1899 \phi .
$$

The numerical values in Eqs. (8) and (9) were determined by the least squares method with the data measured for a general sedan vehicle.

Fig. 5(b) and (c) show the force-stroke(angle) properties of pedal and steering around the rotational axis built in the developed simulator, which were determined based on the measured data. The pedal force-stroke characteristics was modeled by

$$
\tau_{\phi}= \begin{cases}(15 \phi+\dot{\phi}) l_{d} & ((0<\phi<1) \cap(\dot{\phi} \geq 0)) \\ \left(\frac{8}{7} \phi+\dot{\phi}+\frac{97}{7}\right) l_{d} & ((\phi \geq 1) \cap(\dot{\phi} \geq 0)) \\ (9 \phi+\dot{\phi}) l_{d} & ((0<\phi<1) \cap(\dot{\phi}<0)) \\ \left(\frac{8}{7} \phi+\dot{\phi}+\frac{55}{7}\right) l_{d} & ((\phi \geq 1) \cap(\dot{\phi}<0))\end{cases}
$$

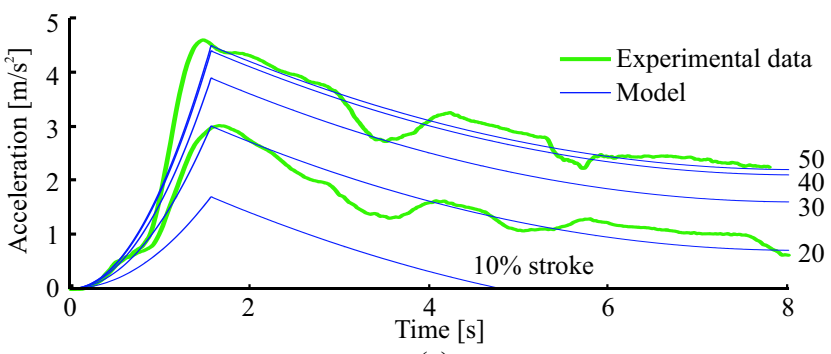

(a)

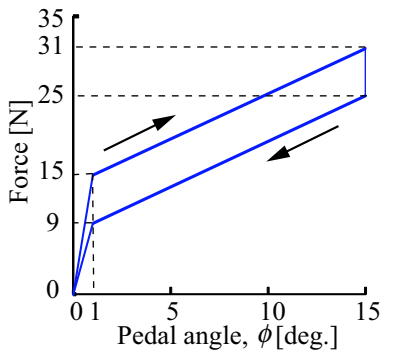

(b)


(d)

Fig. 5. A set of mechanical characteristics of the vehicle.

while the steering dynamics was as

$$
\tau_{\text {steer }}=1.2 \dot{\theta}_{\text {steer }}+6 \theta_{\text {steer }}+0.41 \text {. }
$$

The 1-DOF spring damper system was set at nine points between human body and seat-back and at three points between human body and seat-cushion in this prototype simulator, while the seat stiffness $K_{s} j$ was determined based on the measured data for an actual seat as presented in Fig. 5(d).

\section{Simulation EXPERIMENTS}

\section{A. Driving situation}

The simulation experiments were performed for the curve traveling situation according to the seat heights. The simulation inputs were the driver's ankle-joint torque for pedaling and the angle of the steering wheel as shown in Fig.6. The ankle torque smoothly increased to $4[\mathrm{~N}]$ from $0[\mathrm{~s}]$ to $0.5[\mathrm{~s}]$ and kept constant after that, while the steering angle smoothly rotated to 25 [deg.] from 1 [s] to 3 [s]. 



Fig. 6. The control input of driving interfaces in the simulation.

\section{B. Evaluation and Regulation of operating loads}

Fig. 7(a) presents the changes of driver's posture for the designed simulation inputs according to the two different seat heights (Low and High) by every one second, in which the driver rotates the steering wheel clockwise by the arms and pushes the pedal pad by the right leg. While Fig. 7(b) shows a set of body-pressure distributions at 2 [s], 3 [s] and 4 [s]. The pressure to seat-back in the white frame (around the upside of pelvis) was especially large, and its temporal change was similar to that of vehicle acceleration. It suggests that the driver would receive more information about the vehicle behaviors from the seat-pressure at the upside of pelvis, and this agrees with the opinion of an expert driver. The left side of each color panel became larger as the simulation time was progressed because the change of the driver posture and the increase of centrifugal force were caused by the steering rotation. Also, the pressure to the seat-cushion for the High-condition was sufficiently larger than that for the Low-condition. This corresponds that the lager deformation of the seat-back was occurred under the High-condition.

Fig. 8 shows the operating loads, the joint loads, the perceiving resistance forces, and the seat pressures in the white frame shown in Fig. 7(b). The led/blue solid line indicates the result for Low/High condition, and the broken line in the perceived force denotes the true force provided to the human driver in operating.

The joint load for upper-limb decreases under the Highcondition because the human driver could produce larger torque at the shoulder joint under the specified posture as shown in Fig. 8(a), where the relative angle between the upper body and the upper parts of arms was larger. On the other hand, the joint load for lower-limb increases under the Highcondition as shown in Fig. 8(b) mainly because the inertia effect caused by the vehicle acceleration was larger. Fig. 8(c) shows the time history of the perceived force against the operated force in steering, and indicates that the human driver would feel the different mechanical properties in steering. Note that the perceived steering-force was not affected according to the conditions in this paper since the steering angle was input to the simulator. Fig. 8(d) shows the time history of the perceived pedal-force for the input ankle-torque for the two conditions. Similarly to the steering operations, the driver would feel the different operational resistance according to the seat conditions. These perceptual evaluation results demonstrate that the driving interface should be designed and arranged with well-consideration of human perception properties in the improvement of operational feeling, although another pattern of the simulation inputs is one of main issues
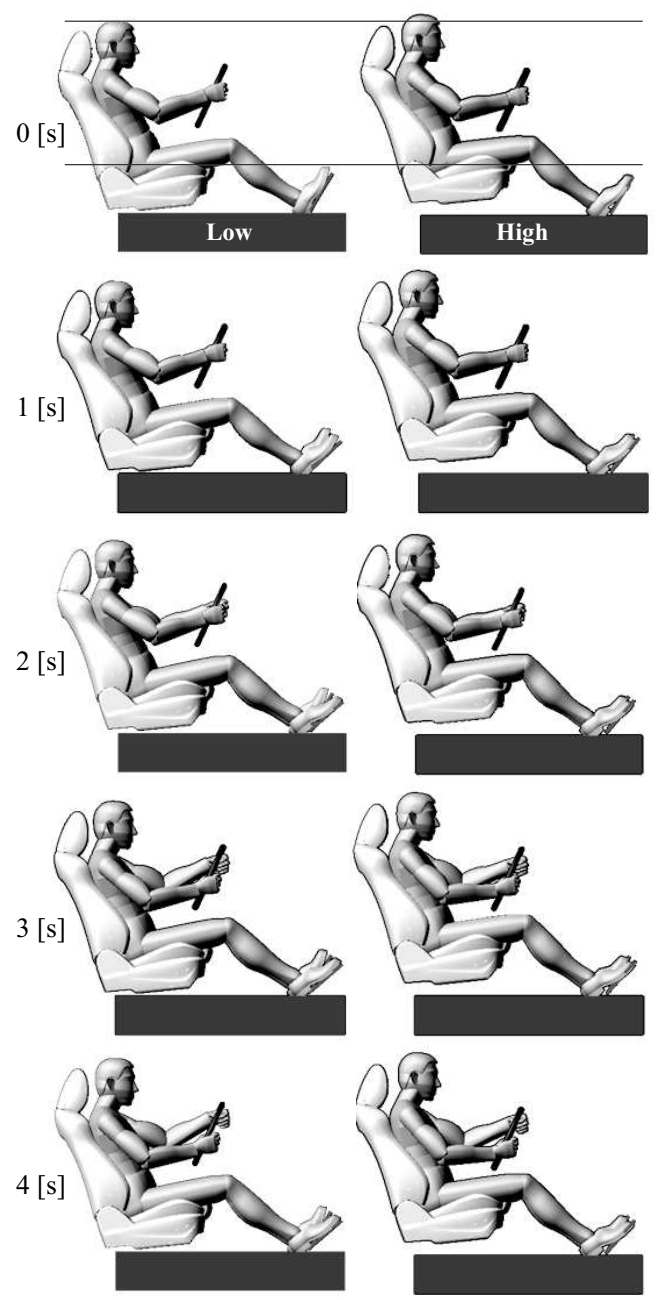

(a)

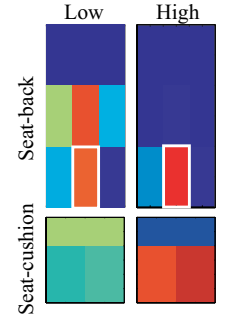

$2[\mathrm{~s}]$



3 [s]



$4[\mathrm{~s}]$
Fig. 7. Changes of driving posture and seat pressure according to the seat hights.

in the future research. Fig. 8(e) shows the time histories of the seat-pressure in the white frame. Since the seat-pressure for the High-condition is much larger than that for the Lowcondition, the High-condition would be better for the human driver to feel vehicular motion in this simulation.

Finally, as a case study, to control operating loads in driving was tried by regulating vehicle characteristics: The goal is to close the operating loads on the High-condition to that on the Low-condition as much as possible. The regulation results show with the green sold line in Fig. 8, where the engine characteristics was 2.1 times, the pedal force-stroke characteristics 


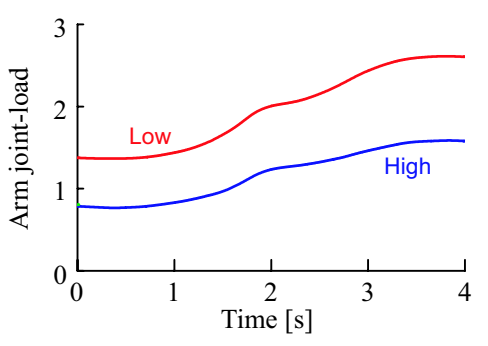

(a)

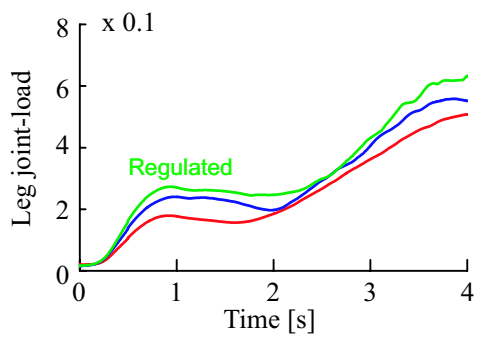

(b)

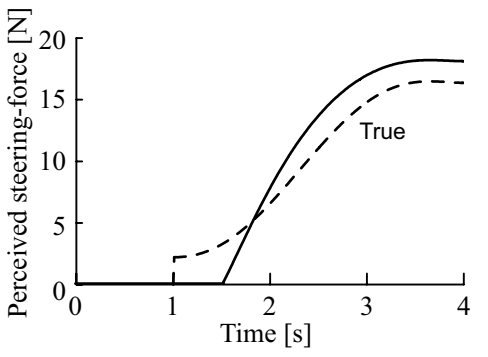

(c)

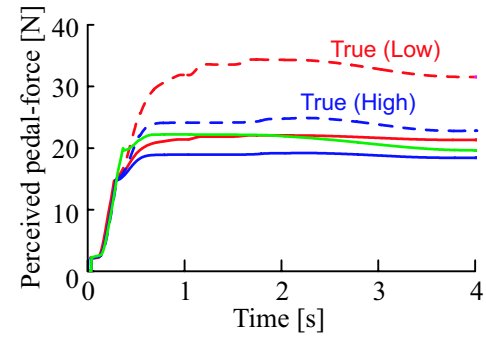

(d)

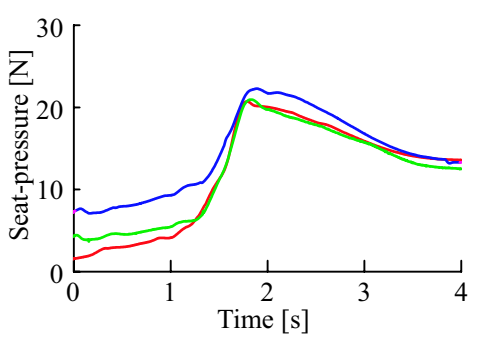

(e)

Fig. 8. Evaluation results of operating loads according to the seat heights in the case of the curve traveling.

was increased $5[\mathrm{~N}]$ and the seat stiffness around the waist was 0.5 times. The joint load for lower limbs does not change significantly, but do close to the result for the Low-condition by regulating engine characteristics and seat viscoelasticity. The obtained results demonstrate that the operating loads could be evaluated and controlled by the developed prototype simulator although the completeness of the simulator needs to improve.

\section{CONCLUSION}

This paper proposed an evaluation methodology for operating loads in vehicle driving based on biomechanical and psychophysical evidences, and developed the prototype simulator by using a big experimental data of human motor properties and force-perception properties. Simulation experiments were performed to evaluate the operating loads (joint-load, seat pressure, and perceived force) according to the different layouts of driving seat in the case of the curve traveling situation. The results indicated that the developed simulator can evaluate operating loads according to the vehicle behavior and the driver posture and can help to improve the driving feel of vehicles. The future research will be directed to enrich the driver database and to install the optimization technique, such as the genetic algorithm, so as to find the suitable vehicle characteristics for comfortable driver feels.

\section{REFERENCES}

[1] S. Park and T. B. Sheridan: "Enhanced Human-Machine Interface in Braking," IEEE Transactions on Systems, Man, and Cybernetics-Part A, Vol. 34, pp. 615-629, 2004.

[2] A. Hirao, S. Kitazaki and N. Yamazaki: "Development of a New Driving Posture for Fatigue Reduction Focused on Biomechanical Loads," Transactions of the Society of Automotive Engineers of Japan, Vol. 39, No. 2, pp. 87-92, 2008 (in Japanese).

[3] Y. Tanaka, N. Yamada, I. Masamori, and T. Tsuji: "Manipulability analysis of lower extremities based on human joint-torque characteristics," Proceedings of the 2nd International Symposium on Measurement, Analysis and Modeling of Human Functions, pp. 261-266, 2004.
[4] Y. Tanaka, N. Yamada, K. Nishikawa, I. Masamori, and T. Tsuji: "Manipulability analysis of human arm movements during the operation of a variable-impedance controlled robot," Proceedings of the 2005 IEEE/RSJ International Conference on Intelligent Robotics and Systems, pp. 3543-3548, 2005.

[5] D. A. Abbink and V. D. Helm: "Force Perception Measurements at the Foot," Proceedings of the 2004 IEEE International Conference on Systems, Man and Cybernetics, Vol. 3, pp. 2525-2529, 2004.

[6] D. A. Abbink, V. D. Helm, and E. R. Boer: "Admittance Measurements of the foot during 'Maintain Position' and 'Relax' Tasks on a Gas Pedal,' Proceedings of the 2004 IEEE International Conference on Systems, Man and Cybernetics, Vol. 3, pp. 2519-2524, 2004.

[7] X. Wang, B. L. Breton-Gadegbeku, and L. Bouzon: "Biomechanical evaluation of the comfort of automobile clutch pedal operation," International Journal of Industrial Ergonomics, Vol. 34, Issue 3, pp. 209-221, 2004.

[8] Y. Tanaka, H. Kaneyuki, T. Tsuji, T. Miyazaki, K. Nishikawa, and T. Nouzawa: "Mechanical and Perceptual Analyses of Human Foot Movements in Pedal Operation," Proceedings of the 2009 IEEE International Conference on Systems, Man, and Cybernetics, pp. 1736-1741, 2009.

[9] K. Takemura, N. Yamada, A. Kishi, T. Nouzawa, Y. Tanaka, Y. Kurita and T. Tsuji: "A Subjective Force Perception Model of Humans and Its Application to a Steering Operation System of a Vehicle," Transactions of the Japan Society of Mechanical Engineers, Vol. 78, No. 795, pp. 64-73, 2012 (in Japanese).

[10] Y. Takeda, Y. Tanaka and T. Tsuji: "Impedance simulator: Analysis of Human Hand Impedance Characteristics" Proceedings of the 2nd International Symposium on Measurement, Analysis and Modeling of Human Functions, pp. 237-247, 2004.

[11] S. S. Stevens: "Psychophysics: Introduction to its Perceptual, Neural, and Social Prospects," Ed. G. Stenens, New York: John Wiley, 1975.

[12] M. Ae: "The inertia parameters of the body for Japanese children and athletes," Japanese Journal of Sports Sciences, Vol. 15, No. 3, pp. 155162, 1996.

[13] Y. Tanaka, Y. Kashiba, N. Yamada, T. Suetomi, K. Nishikawa, T. Nouzawa and T. Tsuji: "Active-Steering Control System Based on Human Hand Impedance Properties," Proceedings of the 2010 IEEE International Conference on Systems, Man, and Cybernetics, pp. 1697-1702, 2010.

[14] Y. Tanaka, T. Onishi, T. Tsuji, N. Yamada, Y. Takeda and I. Masamori: "Analysis and Modeling of Human Impedance Properties for Designing a Human-Machine Control System," Proceedings of the 2007 IEEE International Conference on Robotics and Automation, pp. 3627-3632, 2007. 